\title{
Serotonin receptor agonists in the acute treatment of migraine: a review on their therapeutic potential
}

This article was published in the following Dove Press journal: Journal of Pain Research

\author{
Andrea Negro ${ }^{1,2}$ \\ Angela Koverech ${ }^{2,3}$ \\ Paolo Martelletti ${ }^{1,2}$ \\ 'Department of Clinical and \\ Molecular Medicine, Sapienza \\ University, Rome, Italy; ${ }^{2}$ Department \\ of Internal and Emergency Medicine, \\ Regional Referral Headache Centre, \\ Sant'Andrea Hospital, Rome, Italy; \\ ${ }^{3}$ Department of Physiology and \\ Pharmacology "Vittorio Erspamer", \\ Sapienza University, Rome, Italy
}

\begin{abstract}
Migraine is an important socioeconomic burden and is ranked the sixth cause of years of life lost because of disability in the general population and the third cause of years of life lost in people younger than 50 years. The cornerstone of pharmacological treatment is represented by the acute therapy. The serotonin (5-hydroxytryptamine [5-HT]) receptor subtype $1_{B} / 1_{D}$ agonists, called triptans, are nowadays the first-line acute therapy for patients who experience moderate-to-severe migraine attacks. Unfortunately, a high percentage of patients are not satisfied with this acute treatment, either for lack of response or side effects. Moreover, their mechanism of action based on vasoconstriction makes them unsuitable for patients with previous cardio- and cerebrovascular diseases and for those with uncontrolled hypertension. Since the introduction of triptans, no other acute drug class has passed all developmental stages. The research for a new drug lacking vasoconstrictive effects led to the development of lasmiditan, a highly selective 5-HT1 $1_{\mathrm{F}}$ receptor agonist with minimized interactions with other 5-HT receptor subtypes. Lasmiditan is considered to be the first member of a new drug category, the neurally acting anti-migraine agent (NAAMA). Phase II and III trials had shown superiority compared to placebo and absence of typical triptan-associated adverse events (AEs). Most of the AEs were related to the central nervous system, depending on the high permeability through the blood-brain barrier and mild to moderate severity. The results of ongoing long-term Phase III trials will determine whether lasmiditan will become available in the market, and then active triptan comparator studies will assess patients' preference. Future studies could then explore the safety during pregnancy and breastfeeding or the risk that overuse of lasmiditan leads to medication overuse headache.
\end{abstract}

Keywords: migraine, acute treatment, lasmiditan, 5-HT1 $1_{\mathrm{F}}$ agonists

\section{Introduction}

Migraine is a primary headache disorder characterized by moderate-to-severe headache attacks lasting 4-72 hours with unilateral location and pulsating quality, aggravated by movement or causing avoidance of routine physical activity and associated with nausea and/or vomiting, photophobia, and phonophobia. ${ }^{1}$ Migraine is an important socioeconomic burden and is ranked the sixth cause of years of life lost because of disability in the general population and the third cause of years of life lost in people younger than 50 years. ${ }^{2-5}$

Migraine is an episodic disorder, but its frequency during the lifetime can fluctuate back and forth from a low to a high pattern. Each year, $3 \%$ of patients with episodic migraine develop new-onset chronic migraine (CM), with headache occurring $\geq 15$ days/month (for $>3$ months with at least eight attacks having migraine features). ${ }^{1}$
Correspondence: Andrea Negro

Department of Internal and Emergency Medicine, Regional Referral Headache Centre, Sant'Andrea Hospital, Via di Grottarossa 1035, 00189 Rome, Italy Email andrea.negro@uniromal.it 
When the frequency of the attacks is four or more per month, a preventative therapy with one or more of the following pharmacological classes is required: antihypertensive agents (e.g., $\beta$-blockers, calcium channel blockers, angiotensinconverting enzyme [ACE] inhibitors, aldosterone receptor blockers), antiepileptic drugs (e.g., topiramate, divalproex sodium), and tricyclic antidepressants (e.g., amitriptyline, nortriptyline). ${ }^{6}$ Botox $\AA$ (onabotulinumtoxin $A$ ) is indicated for the prevention of CM.

The cornerstone of pharmacological treatment is represented by the acute therapy, aimed to abort attacks and lead to a prompt relief from pain. Migraine acute therapy is based on nonspecific (analgesics and nonsteroidal anti-inflammatory drugs [NSAIDs]) or specific (triptans and ergot derivatives) drugs. The choice may be based on a stratified care approach (i.e., depending on migraine severity and other clinical factors) or on the step care management (i.e., if the response to analgesics is not sufficient, patients might receive specific drugs). ${ }^{7}$

\section{Current acute treatments}

\section{Triptans (5-HTIB/ID receptor agonists)}

The serotonin (5-hydroxytryptamine [5-HT]) receptor subtype $1 \mathrm{~B} / 1 \mathrm{D}$ agonists (triptans) are nowadays the first-line acute therapy for patients who experience moderate-tosevere migraine attacks. Since the introduction of Sumavel DosePro $®$ (sumatriptan) in 1991, other triptan compounds with improved pharmacokinetic properties, efficacy, and safety were developed. They are more lipophilic than sumatriptan and consequently more capable to penetrate the bloodbrain barrier (BBB), thereby reaching their site of action more readily. Currently, seven triptans are available; in order of release, they are as follows: sumatriptan, zolmitriptan, rizatriptan, naratriptan, eletriptan, almotriptan, and frovatriptan.

All triptans are superior to placebo and can be considered as effective and safe drugs for the vast majority of migraine patients. ${ }^{8}$ Despite a similar molecular structure, each triptan has its own pharmacokinetic and pharmacodynamic profile. Some of them have characteristics similar to sumatriptan, displaying a rapid dose-dependent efficacy with a higher risk of adverse effects (AEs), while others have a slower relieving effect on migraine symptoms but a more prolonged duration of action and less recurrence of migraine attacks.

\section{Safety and contraindications}

Triptans bind mostly to $5-\mathrm{HT} 1_{B}$ and $5-\mathrm{HT} 1_{\mathrm{D}}$ receptors within cerebral blood vessels (endothelium), leading to a rather selective vasoconstriction and inhibiting the release of neurogenic inflammatory mediators such as calcitonin gene-related peptide (CGRP). ${ }^{9}$

The $5-\mathrm{HT} 1_{\mathrm{B}} / 1_{\mathrm{D}}$ receptors are also present on coronary and limb arteries. ${ }^{10,11}$ Accordingly, triptan administration causes a reduction in coronary artery diameter and a brief constriction of limb arteries. ${ }^{11,12}$ Although these minor constrictions are unlikely to cause symptoms and the risk for cardiovascular events is very low in normal patients, triptans may possibly cause ischemia in those with coronary disease. ${ }^{10}$ Rare cases of stroke, myocardial infarction, and arrhythmia have been reported in temporal relation to use of triptans. ${ }^{13-15}$ For this reason, patients with myocardial infarction, coronary artery disease, stroke, uncontrolled hypertension, and vasculitis cannot use triptans. In addition, for patients with hemiplegic migraine or with prolonged migraine aura, as well as for pregnant women, triptans are not entirely recommended. ${ }^{16,17}$

Although they are safe drugs, class-related side effects (e.g., chest tightness and throat discomfort, muscle pain, and paresthesia) are reported by up to $24 \%$ of patients using oral triptans and by $40 \%$ of patients using subcutaneous formulation. ${ }^{16}$ For instance, injectable formulation may be slightly uncomfortable at the site of injection and can potentiate the events known as triptan sensation. ${ }^{18}$

Migraineurs with four or more attacks per months need a preventative treatment. The frequent presence of comorbidities should drive the choice of a preventive drug, in order to reduce the medication intake and increase the efficacy for both the disorders. ${ }^{19}$ Consequently, choosing the safest acute therapy during migraine prophylactic treatment needs pharmacokinetic and pharmacodynamic considerations. ${ }^{20}$ For example, triptans are contraindicated in patients with comorbid depression taking serotonin reuptake inhibitors due to the theoretical possibility of serotonergic syndrome. ${ }^{21}$

\section{Limitations}

An Australian pharmacoepidemiological study showed that total triptan use has increased at an average annual rate of $112 \%$ over the 18 -year period. ${ }^{22}$ Besides, only a minority of migraine patients use the specific antimigraine drug triptans (10\% in Denmark, $17 \%$ in Italy, and $35 \%$ in France). ${ }^{23-25}$ The most probable reason is the relatively low efficacy. Over 35\% of participants in randomized clinical trials (RCTs) do not benefit from administration of triptans, while others complain about their lack of consistency over time. ${ }^{8}$ Likewise, pain freedom rate at 2 hours after administration of triptans ranges from $12 \%$ (frovatriptan $2.5 \mathrm{mg}$ ) to $40 \%$ (rizatriptan $10 \mathrm{mg}$ ). ${ }^{26}$

More indeed, the American Migraine Prevalence and Prevention Study found that $40 \%$ of episodic migraine patients 
have unmet treatment needs, with dissatisfaction with current migraine medication (15\%) and headache-related disability $(19 \%)$ as the most frequent complaints. ${ }^{27}$ Therefore, over the past 5 years, most clinical studies investigated how to improve triptan therapy, increasing efficacy and decreasing adverse reactions. The two principal lines of research were the combination therapy and novel triptan administration routes and devices. ${ }^{28}$

\section{Combination therapy}

Most of the clinical trials studied combinations of triptans with NSAIDs, particularly combinations of sumatriptan and Trixemet ${ }^{\circledR}$ (naproxen). Recent systematic reviews have shown that sumatriptan-naproxen is more effective than placebo and sumatriptan or naproxen alone regarding pain free and pain relief. ${ }^{7,29-32}$ Compared to their usual migraine therapy, patients found the combination of sumatriptannaproxen to be superior in decreasing headache severity, lessening associated symptoms, and providing long-lasting relief with a high rate of complete relief with a single dose (60.04\% of attacks resolved at 2 hours post treatment). ${ }^{33}$ The use of sumatriptan-naproxen is associated with rapid and consistent restoration of patients' functioning, faster recovery time in some measures of cognitive efficiency, and consistent reduction in productivity loss compared to placebo. ${ }^{34,35}$ In general, combination therapy has higher satisfaction ratings. ${ }^{33-35}$

The combination of another triptan, frovatriptan $2.5 \mathrm{mg}$, with two doses of a different NSAID, dexketoprofen 25 and $37.5 \mathrm{mg}$, has been tested for the therapy of acute migraine. ${ }^{36}$ Compared to frovatriptan alone, the group treated with the combination therapy had a significantly higher pain-free rate at 2 and 4 hours and sustained pain-free rate at 24 hours, with no statistically significant difference between participants treated with the dexketoprofen 25 or $37.5 \mathrm{mg}$ combination. ${ }^{36}$ Sustained pain-free rate at 48 hours and recurrence of migraine were similar between the three groups, meaning a lack of improvement with the combination therapy.

Recently, a multicenter, randomized, double-blind trial evaluated the efficacy and safety of the combination of sumatriptan $50 \mathrm{mg}$ plus an antiemetic agent promethazine $25 \mathrm{mg} .{ }^{37}$ Significantly more patients receiving the combination therapy experienced 2- and 4-hour pain-free response and headache improvement response compared to those treated with sumatriptan alone. In addition, the incidence of headache recurrence within 2-48 hours after treatment was lowest in the sumatriptan-promethazine group. However, significant drug-related AEs were more frequent with combination therapy than with sumatriptan alone (somnolence in $32.2 \%$ and $7 \%$ patients and extrapyramidal symptoms in $4.3 \%$ and $0 \%$ patients, respectively), while nausea was more frequent with triptan therapy alone ( $1 \%$ and $8 \%$ patients, respectively).

\section{New formulations and device}

Triptans are generally effective for migraine treatment but share one problem common to all oral drugs, the delayed absorption of the drug depending on the gastric stasis that occurs during the migraine attacks. ${ }^{38,39}$ This issue has led to the development of new administration routes and devices. Two Cochrane database systematic reviews have focused on intranasal and suppository sumatriptan..$^{40,41}$ Both the formulations have been effective in treating the attack, alleviating migraine-associated symptoms, and restoring functional disability.

A randomized, active-comparator, double-dummy, crossover, multi-attack study (COMPASS study) compared the efficacy and tolerability of AVP-825, a bidirectional breathpowered intranasal delivery system containing low-dose (22 mg) sumatriptan powder vs. oral sumatriptan $100 \mathrm{mg} .{ }^{42}$ Rates of pain relief and pain freedom at 2 hours and sustained pain freedom from 2 to 48 hours were similar, but at earlier time point, measured between 15 and 90 minutes, AVP-825 was superior. Nasal discomfort and abnormal taste were more common with AVP-825, while atypical sensation rates were significantly lower than with conventional sumatriptan $100 \mathrm{mg}$. A post hoc analysis found that AVP-825, compared to oral sumatriptan, led to a more rapid early reduction in overall nausea rate during the first hour, a reduced odds of nausea from 30 minutes to 2 hours following treatment, and a reduced risk of treatment-emergent nausea. ${ }^{43}$

Until recently, the subcutaneous administration route was dependent upon a needle-based mechanism. A needle-free subcutaneous sumatriptan is a nitrogen pressure subcutaneous delivery system approved for the acute treatment of migraine and cluster headache in the US and some European countries. ${ }^{44}$ A single dose of needle-free subcutaneous sumatriptan $6 \mathrm{mg}$ demonstrated bioequivalence to a single dose of traditional, needle-based subcutaneous sumatriptan $6 \mathrm{mg}$ when delivered into the abdomen or the thigh, but not into the arm, conferring relief as early as 10 minutes after dosing, whereas oral, rectal, and intranasal routes of administration do so in $\sim 1$ hour. ${ }^{18,45}$

In an open-label, multicenter study, sumatriptan was selfadministered by patients currently treated with triptans and less than very satisfied with their acute migraine therapy. ${ }^{46}$ The efficacy results were consistent with those previously 
observed with needle-based sumatriptan, but participants experienced a statistically significant and clinically relevant increase in satisfaction with therapy and enhanced confidence in treatment after use.

An alternative to current formulations is transdermal drug delivery, particularly iontophoresis. NP10 $1{ }^{\circledR}$ or Zecuity (formerly known as the Zelrix Migraine Patch) is a transdermal sumatriptan iontophoretic patch designed to release $6.5 \mathrm{mg}$ of sumatriptan (comparable to subcutaneous $6 \mathrm{mg}$ ) over 4 hours by using low-level electrical energy to transport sumatriptan across the skin. In double-blind, randomized, placebo-controlled trials, transdermal sumatriptan was superior to placebo but had a generally low clinical efficacy $(18 \%$ pain free rate at 2 hours) compared with conventional oral sumatriptan $50-100 \mathrm{mg} .{ }^{47,48}$ The patch is currently removed from the US market after postmarketing reports of application site reactions described as burns and scars.

\section{CGRP targeting drugs}

So far, no more recent acute antimigraine treatments have succeeded in passing all developmental stages. Smallmolecule CGRP receptor antagonists, the so-called gepants, have been studied since the early 2000s and were promising attack therapies until few years ago. Despite positive Phase II and III trials demonstrating safety and efficacy, their development was terminated. Intravenous olcegepant (BIBN4096BS) was terminated because of difficulty developing an oral formulation, oral telcagepant (MK-0974) and MK-3207 were terminated for transaminase elevation, and BMS-927711, which did not show evidence of hepatotoxicity, was discontinued in 2012 for unknown reasons. ${ }^{49}$

The development of small-molecule CGRP receptor antagonists that seemed abandoned has resumed in 2015 when Allergan purchased the rights of ubrogepant (MK1602) from Merck. In a completed Phase IIb dose-finding study, ubrogepant doses of 25, 50, and $100 \mathrm{mg}$ were superior to placebo for the 2-hour pain freedom end point, showing a dose-dependent response. ${ }^{50}$ Triptan-associated AEs were not observed, and only nausea and dizziness were more common in the ubrogepant group than in the placebo group. Although there was no signal of transaminase elevation, subjects only received a single dose. A second Phase IIb pharmacokinetic study was recently completed, but results have not yet been published. There are two ongoing Phase III RCTs (NCT02867709 and NCT02867709) expected to be completed in March 2018. In both studies, subjects were randomized to treat a single migraine attack either with pla- cebo or with one of two doses of ubrogepant (25 or $50 \mathrm{mg}$ in the first study and 50 or $100 \mathrm{mg}$ in the other study).

\section{Lasmiditan overview \\ Chemistry}

The first 5-HT1 $1_{\mathrm{F}}$ receptor agonist to be tested was LY334370. ${ }^{51}$ This compound, while preserving the typical triptan indole core, showed a high selectivity for $5-\mathrm{HT} 1_{\mathrm{F}}(100$-fold selectivity for $5-\mathrm{HT}_{\mathrm{B}}$ and $5-\mathrm{HT} 1_{\mathrm{D}}$ receptors) and also for $5-\mathrm{HT} 1_{\mathrm{A}}$ receptors. ${ }^{52}$ Even so, its development was discontinued because of long-term safety concerns in animals. ${ }^{53}$ Therefore, a new selective 5-HT1 $1_{\mathrm{F}}$ receptor agonist was developed changing the chemical structure of LY334370 by replacing the indole moiety with a pyridinoyl-piperidine scaffold. Lasmiditan, formerly known as COL-144 and LY573144, is a novel 5-HT receptor agonist with high affinity and selectivity for the $5-\mathrm{HT} 1_{\mathrm{F}}$ receptor. Its chemical structure is more different from triptans than its predecessor LY334370 and accounts for its inclusion in a new drug class called ditans (Figure 1). ${ }^{54}$

\section{Pharmacodynamics}

LY334370 showed relative selectivity for $5-\mathrm{HT} 1_{\mathrm{F}}$ receptor (100-fold selectivity for 5-HT1 $1_{\mathrm{F}}$ receptors than for 5-HT1 $1_{\mathrm{B}}$ and $5-\mathrm{HT} 1_{\mathrm{D}}$ receptors) and a substantial affinity for the $5-\mathrm{HT} 1_{\mathrm{A}}$ receptor. ${ }^{52}$ However, a concern rose about the possibility that plasma levels in the therapeutic range might be high enough to activate vascular 5-HT1 $1_{\mathrm{B}}$ receptors. Lasmiditan met the goal of developing a highly selective agonist for the 5-HT1 $1_{\mathrm{F}}$ receptor, structurally different from the triptans, with a greater 5-HT1 $1_{\mathrm{F}}$ receptor selectivity than LY334370 and with minimized interactions with $5-\mathrm{HT} 1_{A}, 5-\mathrm{HT} 1_{B}$, and $5-\mathrm{HT} 1_{D}$ receptors to avoid potential concerns arising for its predecessor. In vitro binding studies showed a 450-fold higher selectivity and higher affinity for $5-\mathrm{HT} 1_{\mathrm{F}}$ receptors than for $5-\mathrm{HT} 1_{\mathrm{A}}, 5-\mathrm{HT} 1_{\mathrm{B}}$, and $5-\mathrm{HT} 1_{\mathrm{D}}$ receptors, and a low cross-reactivity with other members of the 5-HT1 receptor family, even if all of them are structurally homologous. ${ }^{55}$ Moreover, lasmiditan showed no significant affinity across a panel of receptors that are known to regulate vascular tone, the monoaminergic subtypes. ${ }^{55}$

In addition to targeting peripheral $5-\mathrm{HT} 1_{\mathrm{F}}$ receptors, lasmiditan, being a high lipophilic substance, is able to penetrate the BBB and may act on centrally located receptors. ${ }^{55}$ This hypothesis is supported by the results of two animal models of migraine involving electrical stimulation of the trigeminal ganglion that are considered predictive for drug efficacy in acute migraine treatment. Oral administration of lasmiditan at a dose $\geq 3 \mathrm{mg} / \mathrm{kg}$ blocked neurogenic inflammation through inhibition 
A

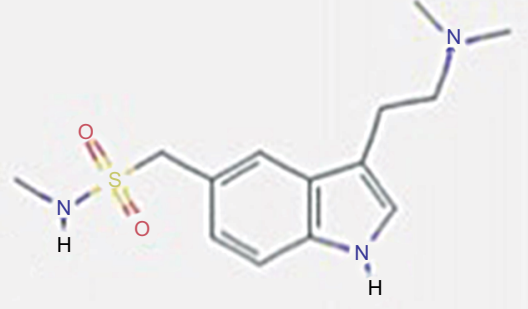

1-[3-[2-(Dimethylamino)ethyl]-1H-indol-5-yl]- $N$-methylmethanesulfonamide

C

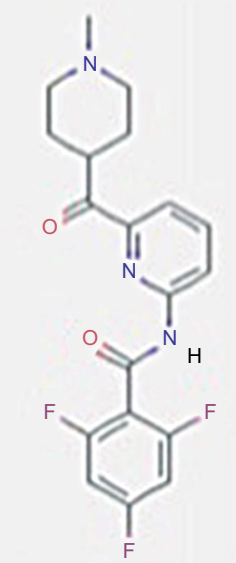

1-[3-[2-(Dimethylamino)ethyl]-1H-indol-5-yl]- $N$-methylmethanesulfonamide
B

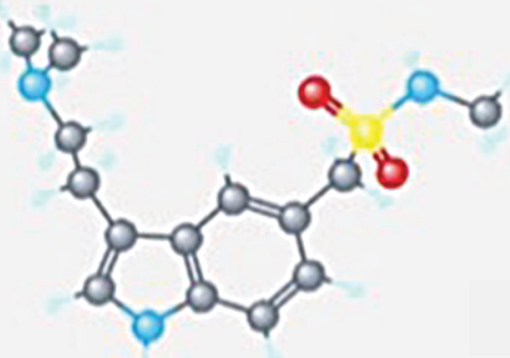

D

Lasmiditan

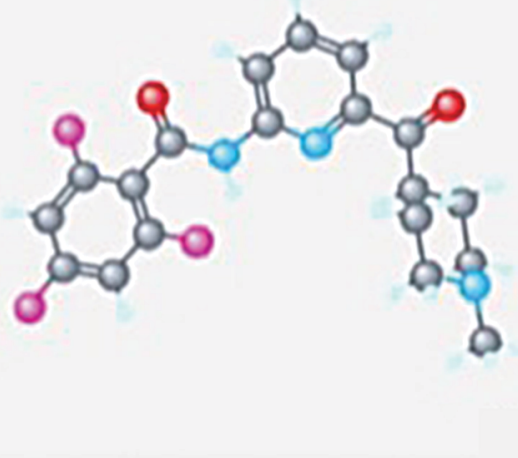

Figure I Comparison of chemical structure of sumatriptan and lasmiditan: 2D structure (A and $\mathbf{C})$ and 3D conformer (B and $\mathbf{D})$.

of dural plasma protein extravasation and reduced expression of a proto-oncogene (c-Fos) in the trigeminal nucleus caudalis. ${ }^{55}$

The selective functional activity of lasmiditan was demonstrated in vitro in cells expressing the human isoforms of the subtypes of the 5-HT1 receptors using a standard radioligand-binding assay ([35 S]-GTP $\gamma \mathrm{S}$ ). While triptans had high potency and efficacy at human $5-\mathrm{HT} 1_{\mathrm{B}}$ and $5-\mathrm{HT} 1_{\mathrm{D}}$ receptors, lasmiditan showed low potency at the vasoconstrictor 5-HT1 ${ }_{\mathrm{B}}$ receptors. ${ }^{55}$ Moreover, lasmiditan (in doses up to $100 \mu \mathrm{M}$ ) did not cause vessel contraction in the rabbit saphenous vein, which is a surrogate model that predicts vasoconstriction in human coronary arteries.$^{55}$ In contrast, sumatriptan led to a $50 \%$ vasoconstriction.

Because of the high selectivity for the $5-\mathrm{HT} 1_{\mathrm{F}}$ receptors and the absence of vasoconstrictive effects, $5-\mathrm{HT} 1_{\mathrm{F}}$ receptor agonists are considered a new drug category, the neurally acting anti-migraine agent (NAAMA), acting through a unique and promising new antimigraine mechanism. ${ }^{54}$

\section{Pharmacokinetics and metabolism}

Lasmiditan has a mean oral bioavailability reported to be $\sim 40 \%$, and the maximum serum concentration of lasmiditan is reached between 1.5 and 2.5 hours $\left(\mathrm{T}_{\max }\right)$ after oral administration of 50-400 mg. ${ }^{56}$ There are no available data about the possible influence of gender and age on these parameters. A recent randomized, open-label study evaluated the relative bioavailability of lasmiditan (Table 1). ${ }^{57}$ The maximum serum concentration $\left(C_{\max }\right)$, $\mathrm{T}_{\text {max }}$, and the area under the curve (AUC) of lasmiditan $200 \mathrm{mg}$ under fasted and fed conditions were evaluated in 30 healthy subjects. $C_{\max }$ was, respectively, $322.8 \pm 122.0$ and $394.7 \pm 167.8 \mathrm{ng} / \mathrm{mL}$ (mean $\pm \mathrm{SD})$; $\mathrm{T}_{\max }$ was, respectively, $1.5 \pm 1.0$ and $2.5 \pm 1.0$ hours (mean $\pm \mathrm{SD}$ ); and $\mathrm{AUC}_{0-\mathrm{t}}$ was, respectively, $1892 \pm 746.0$ and $2244 \pm 926.2 \mathrm{ng} \times \mathrm{h} / \mathrm{mL}$ (mean $\pm \mathrm{SD}$ ). There are no data about lasmiditan half-life $\left(\mathrm{t}_{1 / 2}\right)$ and metabolism; therefore, the existence of active and/ or inactive metabolites is unknown. 
Table I Summary of pharmacokinetic characteristics of serotonin receptor agonists

\begin{tabular}{|c|c|c|c|c|c|c|}
\hline Compound & Dosing (mg) & $\mathbf{T}_{1 / 2}$ (hours) & Bioavailability (\%) & $\mathbf{T}_{\max }$ (hours) & $C_{\max }(\mathrm{ng} / \mathrm{mL})$ & $\operatorname{AUC}(0, \infty)(\mathrm{ng} / \mathrm{mL} \times \mathrm{h})$ \\
\hline \multirow[t]{3}{*}{ Sumatriptan } & Oral 50 & 2 & 14 & 2.5 & $27-30$ & 118 \\
\hline & Oral 100 & 2 & 24 & 2.5 & 54 & 158 \\
\hline & NS 20 & $1.2-3$ & 17 & 1 & 13 & 48 \\
\hline \multirow[t]{2}{*}{ Lasmiditan } & Oral 200 & - & - & $1.5 \pm 1.0^{\mathrm{a}}$ & $322.8 \pm 122.0^{\mathrm{a}}$ & $1892 \pm 746.0^{\mathrm{a}}$ \\
\hline & & & & $2.5 \pm 1.0^{\mathrm{b}}$ & $394.7 \pm 167.8^{b}$ & $2244 \pm 926.2^{\mathrm{b}}$ \\
\hline
\end{tabular}

Notes: $T_{1 / 2}$, elimination half-life; $T_{\max }$, time to maximum concentration; $C_{\max }$, maximum observed concentration. ${ }^{\text {a }}$ Fasted condition. ${ }^{b}$ Fed condition. Data from Lionetto et al ${ }^{18}$ and Food-Effect Study in Healthy Subjects. ${ }^{57}$

Abbreviations: AUC, area under the curve; NS, nasal spray.

\section{Phase I clinical trials}

Five Phase I studies have been conducted for lasmiditan (four for the oral formulation and one for the intravenous formulation). ${ }^{58}$ Peer-reviewed publications for the Phase I trials do not exist. In each of these studies, $40-55$ healthy subjects were evaluated to test the safety, tolerability, pharmacodynamics, and pharmacokinetics of lasmiditan.

The first Phase I trial was performed in 2003 to evaluate safety, tolerability, and pharmacokinetics of intravenous administration of lasmiditan (no published data available). In 2008, two Phase I trials (COL MIG-102 and COL MIG-103) tested different oral formulations (oral solution, oral tablet, and sublingual) to evaluate their safety, tolerability, bioavailability, and other pharmacokinetic parameters. Oral formulations demonstrated to reach plasma levels associated with efficacy of the intravenous formulation without severe AEs. ${ }^{59}$ The oral formulation dose at least effective as sumatriptan in acute migraine treatment was calculated to be $\geq 170 \mathrm{mg} .{ }^{60}$

In 2011, the Phase I study COL MIG-105 evaluated the cardiac safety of lasmiditan. This randomized, double-blind study compared the effects on cardiac depolarization and repolarization duration and other cardiac safety parameters of oral lasmiditan (100 and $400 \mathrm{mg}$ ) with those of the antibiotic moxifloxacin (400 mg) and placebo. Lasmiditan at both doses did not cause QT prolongation compared to moxifloxacin that led to QT prolongation as seen in other published studies. ${ }^{61}$ Moreover, no arrhythmia or any proarrhythmic effects were reported with lasmiditan.

The last Phase I study investigated the effects of the fed and fasted states on pharmacokinetic parameters $\left(C_{\max }, \mathrm{T}_{\max }\right.$, AUC) and AEs. ${ }^{57}$ The fed condition was associated with an increase in $C_{\max }, \mathrm{T}_{\max }$, and AUC and a lower rate of mild AEs.

Several Phase I clinical trials are currently undergoing, while others have been recently completed, but the results are not yet available (Table 2).

\section{Phase II clinical trials}

Two Phase II trials were conducted in 2007 and 2009 for the intravenous formulation and the oral formulation, respectively. ${ }^{56,62}$
The first study was a proof-of-concept and dose-finding, multicenter, randomized, double-blind, placebo-controlled trial that enrolled 130 migraine patients with a migraine history of $\geq 1$ year and not taking prophylactic medication. ${ }^{62}$ The adaptive treatment design of the study allowed the up- or down-titration of the study drug (either lasmiditan i.v. in doses of $2.5-45 \mathrm{mg}$ or placebo) according to efficacy and AEs. The primary efficacy measure was headache response (improvement from moderate or severe headache to mild or no headache) at 2 hours. Better response was observed in the groups receiving lasmiditan compared to placebo $(54.2 \%-75 \%$ vs. $45.2 \%$ ) with a significant dose-response relationship with increasing doses of lasmiditan and with doses of $\geq 20 \mathrm{mg}$ being more effective. For lasmiditan $20 \mathrm{mg}$, the headache response was $64 \%$ with a therapeutic gain (percentage difference between active drug and placebo) of 19\%. Accordingly, the use of rescue medication decreased while patients' global impression improved significantly with the increasing dose. The onset of pain relief occurred in 20-40 minutes.

The second Phase II study was a dose-ranging, multicenter, randomized, double-blind, placebo-controlled trial that enrolled 534 migraine patients with a migraine history of $\geq 1$ year and not taking prophylactic medication. ${ }^{56}$ Patients were randomized to treat one migraine attack with a rapid disintegration tablet of lasmiditan (in doses of 50, 100, 200, and $400 \mathrm{mg}$ ) or placebo in a 1:1:1:1:1 ratio. Every lasmidi$\tan$ dose was superior to placebo for the primary efficacy measure (headache response at 2 hours) with a significant linear dose-response association. After 1 hour, all the doses, except the $50 \mathrm{mg}$ dose, were superior to placebo, and the $400 \mathrm{mg}$ dose significantly reduced headache severity starting as early as 30 minutes, and after 90 minutes, all groups reached statistical significance vs. placebo. The therapeutic gains for headache responses at 2 hours were 18\%, 38\%, $29 \%$, and $39 \%$ for $50,100,200$, and $400 \mathrm{mg}$ of lasmiditan, respectively, compared to placebo. The therapeutic gain of lasmiditan $400 \mathrm{mg}$ (39\%) was higher than that of the intravenous $20 \mathrm{mg}$, probably because of the dose difference (oral $400 \mathrm{mg}$ corresponds to i.v. dose of $\sim 160 \mathrm{mg}$ ). ${ }^{26}$ The headache 
Table 2 Summary of completed and ongoing Phase I clinical trials on lasmiditan

\begin{tabular}{llll}
\hline ID number & Study name & Primary outcome measures & Completion date \\
\hline NCT03247790 & $\begin{array}{l}\text { An open-label, two-period study to evaluate the } \\
\text { pharmacokinetics of lasmiditan in migraineurs }\end{array}$ & $\begin{array}{l}C_{\max } \text { of lasmiditan in each period } \\
\text { AUC }(0-\infty) \text { of lasmiditan in each period }\end{array}$ & March 2018
\end{tabular}
during acute migraine attacks and during inter-ictal periods

NCT03270644 Effect of lasmiditan on the heart rate and blood pressure in healthy subjects receiving oral doses of propranolol

NCT03182920 Effect of age on the pharmacokinetics, safety, and tolerability of lasmiditan in healthy subjects

NCT03040362 A Phase I study to investigate the absorption, metabolism, and excretion of [14C]-lasmiditan following single oral dose administration in healthy male and female subjects

NCT03009162 A Phase I, multicenter, open-label, parallel-group adaptive pharmacokinetic single dose study of oral lasmiditan in subjects with normal and impaired renal function

\section{NCT03308669 Safety, tolerability, and pharmacokinetics of} lasmiditan when coadministered with topiramate in healthy subjects

NCT033I04II A randomized, double-blind, four-period, crossover study to evaluate the cardiovascular effect of single oral doses of lasmiditan when coadministered with single oral doses of sumatriptan in healthy subjects

NCT03286218 A randomized, subject- and investigator-blind, placebo- and active-controlled study to assess the abuse potential of lasmiditan

NCT032520I5 Multiple-ascending dose, safety, tolerability, pharmacokinetic, and drug-drug interaction study of lasmiditan

NCT03076970 A randomized, double-blind, three period, crossover study to evaluate the effect of single oral doses of lasmiditan when coadministered with single oral doses of sumatriptan (Imitrex) in healthy male and female subjects

NCT03040479 A Phase I, multicenter, open-label, parallelgroup, pharmacokinetic single dose study of oral lasmiditan in subjects with normal and impaired hepatic function

NCT030I2334 A Phase I, randomized, double-blind, placebocontrolled, five-period, cross-over study assessing the effects of lasmiditan on simulated driving performance in normal healthy volunteers
Change in mean hourly heart rate as determined

by Holter ambulatory monitoring

$C_{\max }$ of major lasmiditan metabolites in each period

AUC $(0-\infty)$ of major lasmiditan metabolites in each period

$C_{\max }$ based on plasma concentrations of lasmiditan $\mathrm{T}_{\text {max }}$ based on plasma concentrations of lasmiditan AUC from hour 0 to the last measurable concentration based on plasma concentrations of lasmiditan

AUC $(0-\infty)$ of total radioactivity in blood/AUC

$(0-\infty)$ of total radioactivity in plasma

AUC $(0-\infty)$ of lasmiditan in plasma/AUC $(0-\infty)$ of total radioactivity in plasma

$C_{\max }$ based on plasma concentrations of lasmiditan $\mathrm{T}_{\text {max }}^{\text {max }}$ based on plasma concentrations of lasmiditan AUC $(0-\infty)$

Amount excreted in urine

Fraction of dose excreted in urine

Renal clearance

Number of participants with one or more SAEs considered by the investigator to be related to study drug administration

SBP per 24-hour ABPM

Change from baseline in $E_{\max }$ of bipolar drug liking VAS scores

Number of participants with one or more SAEs considered by the investigator to be related to study drug administration

Change from pre dose to 24 hours in vital signs

Change from pre dose to 24 hours in ECGs

AEs

$C_{\max }$ based on plasma concentrations of lasmiditan $\mathrm{T}_{\text {max }}$ based on plasma concentrations of lasmiditan AUC $(0-\infty)$

Apparent elimination rate constant

Terminal elimination half-life

Simulated driving performance in healthy subjects as measured by SDLP using the CRCDS-MiniSim
November 2017

August 2017

May 2017

June 2017

December 2017

December 2017

November 2017

January 2018

April 2017

July 2017

June 2017

Notes: $C_{\text {max }}$ maximum observed concentration; AUC $(0-\infty)$, area under the concentration vs. time curve from zero to infinity; $T_{\max }$, time to maximum concentration; $E_{\max }$, maximal effect score. Data from clinicaltrials.gov.

Abbreviations: SAE, serious adverse event; SBP, systolic blood pressure; ABPM, ambulatory blood pressure monitoring; VAS, visual analog scale; ECG, electrocardiogram; AE, adverse event; SDLP, standard deviation of lateral position; CRCDS-MiniSim, Cognitive Research Corporation's driving simulator-MiniSim. 
response with $100 \mathrm{mg}$ was superior to $200 \mathrm{mg}$ and was similar to $400 \mathrm{mg}$. The reason may depend on the small sample sizes and the random variation in severity and response of migraine attacks. The therapeutic gains for headache responses of lasmiditan $100 \mathrm{mg}(38 \%)$ and $400 \mathrm{mg}(39 \%)$ are in the same range of oral triptans. ${ }^{8}$ Lasmiditan doses of 200 and $400 \mathrm{mg}$ were superior to placebo ( $19 \%$ and $28 \%$ vs. $7.4 \%$ ) for pain free at 2 hours (secondary end point), but lasmiditan $100 \mathrm{mg}$ (14\%) was not. Both doses of lasmiditan, 100 and $400 \mathrm{mg}$, were superior to placebo for improvements in headache severity, clinical disability, headache recurrence within 24 hours, and patients' global impression of treatment.

\section{Phase III clinical trials}

Currently, two Phase III clinical trials and one long-term, open-label study are ongoing. ${ }^{63-65}$

The first trial is a randomized, double-blind, placebocontrolled parallel group study (COL MIG-301 or SAMURAI, ID number NCT02439320) evaluating the efficacy of two doses of lasmiditan (100 and $200 \mathrm{mg}$ ) for the outpatient treatment of one migraine attack. A second dose within 24 hours was allowed for rescue or recurrence of migraine. ${ }^{63}$ For this study, 2232 patients older than 18 years (with no upper age limit) with episodic disabling migraine (assessed by the Migraine Disability Assessment score $\geq 11$ ) were enrolled. The primary and secondary end points were the proportion of subjects with pain-free headache and the proportion of subjects who were free of most bothersome migraine-associated symptoms at 2 hours post dose. The most bothersome migraine-associated symptom (identified by the subjects among nausea, photophobia, and phonophobia) is a new end point never used before in migraine therapy. Other outcome measures were headache relief (at 2 hours); use of rescue medication (at 2 hours and 2-24 hours); headache recurrence (within 48 hours); proportion of patients who were free of nausea, photophobia, and phonophobia (at 2 hours); AEs (up to 11 weeks); and health care resource utilization (6 months prior to enter in the study compared with its use during the time on study). Both doses showed superiority compared to placebo for the primary and several secondary end points with statistical significance.

The second trial is a randomized, double-blind, placebocontrolled parallel group study (COL MIG-302 or SPARTAN, ID number NCT02605174) evaluating the efficacy of three doses of lasmiditan $(50,100$, and $200 \mathrm{mg})$ for the outpatient treatment of one migraine attack. ${ }^{64}$ The characteristics of the enrolled population (3007 subjects), the design, and the end points of this study were same as of the SAMURAI trial.
The long-term, open-label trial of lasmiditan (COL MIG305 or GLADIATOR, ID number NCT02565186) started in October 2015 and is estimated to end in May $2018 .^{65}$ This is a prospective, randomized, open-label study of subjects with migraine who have completed SAMURAI or SPARTAN. The study is designed to evaluate the safety and tolerability (primary end point) of long-term (12 months) intermittent use of lasmiditan 100 and $200 \mathrm{mg}$ as the first dose and the second dose for the acute treatment of migraine. Long-term efficacy (secondary end point) will also be evaluated, as well as the 12-month health care resource utilization.

\section{Clinical safety and tolerability}

In all studies, lasmiditan did not show the usual side effects of triptans. Typical triptan-associated AEs (chest or neck pain, tightness, or heaviness) were rare and with a rate similar to the placebo-treated groups. ${ }^{56,62}$ This finding was expected considering the different chemical structure of lasmiditan.

Most importantly, cardiac safety of lasmiditan was confirmed as no arrhythmia or any proarrhythmic effects were observed. ${ }^{61}$ No pathological abnormalities of any safety parameters (i.e., vital parameters, 12-lead electrocardiogram [ECG], hematology, biochemistry, and urine analysis) were reported following the administration of lasmiditan, for both the intravenous and oral formulations (all doses up to $400 \mathrm{mg}$ ). ${ }^{56,62}$

However, this selective $5-\mathrm{HT} 1_{\mathrm{F}}$ receptor agonist showed a high incidence of the central nervous system (CNS)-related AEs, probably because of the high CNS permeability through the BBB. In one Phase I study, the most common side effect was somnolence, ${ }^{58}$ while in another study, drowsiness, dizziness, and paresthesia were reported as the most frequent AEs with the maximum dose of $400 \mathrm{mg} .{ }^{59}$ In the first Phase II trial with the intravenous formulation $(20 \mathrm{mg}), 65 \%$ of subjects on lasmiditan and $43 \%$ on placebo reported mild AEs, and the most common were paresthesia, dizziness, and limb heaviness. ${ }^{62}$ The rate of placebo-subtracted AEs for lasmiditan was $25 \%$.

The larger human safety and tolerability data came from the oral lasmiditan Phase II study (Table 3). ${ }^{56}$ AEs of dizziness, paresthesia, vertigo, fatigue, and somnolence were reported by $22 \%$ of the patients receiving placebo and, in a dose-dependent manner, by $65 \%, 72 \%, 86 \%$, and $84 \%$ of patients receiving lasmiditan $(50,100,200$, and $400 \mathrm{mg}$, respectively). The rate of placebo-subtracted AEs was 50\% for oral lasmiditan $100 \mathrm{mg}$ and was $66 \%$ for lasmiditan $400 \mathrm{mg}$. Most AEs were mild. However, with lasmiditan $100 \mathrm{mg}$, moderate and severe AEs were reported by $46 \%$ and $27 \%$ of patients. At the dose of $400 \mathrm{mg}, 60 \%$ of subjects 
Table 3 Treatment-emergent and severe AEs after placebo and oral lasmiditan (50-400 mg)

\begin{tabular}{|c|c|c|c|c|c|c|c|c|c|c|}
\hline \multirow[t]{2}{*}{ AEs } & \multicolumn{2}{|c|}{$\begin{array}{l}\text { Placebo } \\
\text { (n=19/86; 22\%) }\end{array}$} & \multicolumn{2}{|c|}{$\begin{array}{l}\text { Lasmiditan } 50 \mathrm{mg} \\
(\mathrm{n}=53 / 82 ; 65 \%)\end{array}$} & \multicolumn{2}{|c|}{$\begin{array}{l}\text { Lasmiditan } 100 \mathrm{mg} \\
(\mathrm{n}=59 / 82 ; 72 \%)\end{array}$} & \multicolumn{2}{|c|}{$\begin{array}{l}\text { Lasmiditan } 200 \mathrm{mg} \\
(\mathrm{n}=6 \mathrm{I} / 7 \mathrm{I} ; 86 \%)\end{array}$} & \multicolumn{2}{|c|}{$\begin{array}{l}\text { Lasmiditan } 400 \mathrm{mg} \\
(\mathrm{n}=59 / 70 ; 84 \%)\end{array}$} \\
\hline & $\begin{array}{l}\text { Treatment } \\
\text { emergent }\end{array}$ & Severe & $\begin{array}{l}\text { Treatment } \\
\text { emergent }\end{array}$ & Severe & $\begin{array}{l}\text { Treatment } \\
\text { emergent }\end{array}$ & Severe & $\begin{array}{l}\text { Treatment } \\
\text { emergent }\end{array}$ & Severe & $\begin{array}{l}\text { Treatment } \\
\text { emergent }\end{array}$ & Severe \\
\hline Dizziness & $0(0 \%)$ & $0(0 \%)$ & $19(23 \%)$ & $\mathrm{I}(\mathrm{I} \%)$ & $21(26 \%)$ & $8(10 \%)$ & $27(38 \%)$ & II (I5\%) & $26(37 \%)$ & $12(17 \%)$ \\
\hline Fatigue & $2(2 \%)$ & I (I\%) & $10(12 \%)$ & $5(6 \%)$ & 17 (2।\%) & 7 (9\%) & 15 (2I\%) & II (I5\%) & 16 (23\%) & $7(10 \%)$ \\
\hline Vertigo & I (I\%) & $0(0 \%)$ & $8(10 \%)$ & I (I\%) & $12(15 \%)$ & 3 (4\%) & $12(17 \%)$ & $3(4 \%)$ & $16(23 \%)$ & 7 (10\%) \\
\hline Somnolence & $2(2 \%)$ & I (I\%) & 8 (10\%) & $3(4 \%)$ & 10 (12\%) & $2(2 \%)$ & $8(11 \%)$ & $2(3 \%)$ & $8(11 \%)$ & $2(3 \%)$ \\
\hline Paresthesia & $2(2 \%)$ & $0(0 \%)$ & $2(2 \%)$ & I (I\%) & $9(11 \%)$ & $2(2 \%)$ & 12 (I7\%) & $4(6 \%)$ & I4 (20\%) & $5(7 \%)$ \\
\hline Nausea & $0(0 \%)$ & $0(0 \%)$ & $4(5 \%)$ & $2(2 \%)$ & $8(10 \%)$ & $0(0 \%)$ & $2(3 \%)$ & I (I\%) & $5(7 \%)$ & $0(0 \%)$ \\
\hline $\begin{array}{l}\text { Sensation of } \\
\text { heaviness }\end{array}$ & I (I\%) & I (I\%) & $4(5 \%)$ & $3(4 \%)$ & $4(5 \%)$ & I (I\%) & $7(10 \%)$ & $2(3 \%)$ & $5(7 \%)$ & $3(4 \%)$ \\
\hline
\end{tabular}

Note: The first row reports the occurrence rate (\%) of treatment-emergent events calculated as the ratio between subjects experiencing an $A E$ ( $n$ ) and the total population in each treatment group. Modified from Lancet Neurol, I I (5) Farkkila M, Diener HC, Geraud G, et al, Efficacy and tolerability of lasmiditan, an oral 5-HT(IF) receptor agonist, for the acute treatment of migraine: a phase 2 randomised, placebo-controlled, parallel-group, dose-ranging study, 405-4I3, Copyright (20I2), with permission from Elsevier. ${ }^{56}$ Abbreviation: $A E$, adverse event.

reported moderate AEs and $44 \%$ of subjects reported severe AEs. Thus, almost one-third of patients experienced severe CNS-related AEs even with the $100 \mathrm{mg}$ dose. A 46-year-old woman had an overnight hospital admission for moderate dizziness occurring 30 minutes after taking $200 \mathrm{mg}$ of lasmiditan. ECGs showed sinus bradycardia 1.5 and 4 hours after drug intake but no other abnormalities. She received a saline infusion and had complete recovery by the next day. The AE was classified as serious.

In the first Phase III trial (SAMURAI study), lasmiditan was tolerated and, as in the Phase II studies, most AEs were related to the CNS or the vestibular system. ${ }^{66}$ The most common side effect was dizziness ( $11.9 \%$ and $15.4 \%$ for lasmidi$\tan 100$ and $200 \mathrm{mg}$, respectively, vs. 3.1\% for placebo). Other common AEs were paresthesia, nausea, somnolence, and fatigue. AEs were reported to be mild or moderate in $91 \%$ of the cases, and some of them (dizziness and vertigo) occurred less frequently than those observed in the Phase II trials.

\section{Migraine pathophysiology: how does an acute treatment work?}

The pathophysiology of migraine still remains not completely clear, but the current view considers this primary headache as a disorder in which the brain, meningeal blood vessels, and the trigeminal nerve system play a crucial role. ${ }^{67}$ The current standard pharmacotherapies have been developed when migraine was believed to be primarily a vascular disease depending on the abnormal vasodilation of intracranial vessels. ${ }^{6}{ }^{6}$ Hence, it was supposed that constriction of dilated cerebral blood vessels could block a migraine attack.

Although the vascular hypothesis was reinforced by the efficacy of 5-HT1 $1_{B}$ receptor agonists (triptans), which act as potent cerebral vasoconstrictors, it has been overtaken by the neurogenic hypothesis. Recent experimental evidences suggest that migraine is primarily a neuronal disease. ${ }^{67,69,70}$ According to this model, cranial vasodilation is not the primum movens but only an epiphenomenon in migraine pathophysiology. The primary cause is supposed to be the activation and sensitization of the trigeminal nerve system innervating the large vessels in the meninges. ${ }^{71}$ These processes lead to neuropeptide release, particularly CGRP, which is a well-known vasodilatator, and a subsequent increase in vessel diameter and blood flow in the meninges and cortex. In the cascade of events, the following excitation of secondary and tertiary central trigeminal neurons may activate cortical and subcortical areas, giving a reason to the pain and other typical migraine symptoms. ${ }^{72}$

Triptans may act also through neural mechanisms, more likely inhibiting neuropeptide release (i.e., CGRP) from trigeminal neurons and consequently blocking transmission of pain signals within the trigeminal cervical complex. ${ }^{73}$ The neurogenic hypothesis implies the possibility that vasoconstriction is not essential for antimigraine therapy and that migraine may be aborted inhibiting central or peripheral nervous system mechanisms. In this view, CGRP receptor antagonists (gepants) seemed to be promising alternatives to triptans, but, even if effective, their development was interrupted because of the concern regarding hepatotoxicity. ${ }^{74}$

Recently, 5-HT1 $1_{\mathrm{F}}$ receptors, expressed by trigeminal neurons, trigeminal ganglion, and trigeminal nucleus caudalis and lacking vasoconstrictive effects, were thought as an ideal target for migraine abortive drug development. ${ }^{75,76}$ Specifically, lasmiditan has a peculiar and unique chemical structure that avoids typical triptans-related side effects (e.g., neck, jaw, and chest symptoms) and makes it able to cross the $\mathrm{BBB}$ and act both centrally on trigeminal neurons and 
peripherally on primary trigeminal afferents and cell bodies within the trigeminal ganglion. ${ }^{77}$

In Phase II and III trials, lasmiditan proved to be an effective acute treatment for migraine attacks with an AE profile exclusively related to $\mathrm{CNS}$, depending on its penetration through the BBB. By stimulation of the 5-HT1 $1_{\mathrm{F}}$ receptors, lasmiditan may inhibit central and peripheral neuronal activities and the neuropeptide release (e.g., CGRP), thereby terminating acute migraine. ${ }^{75,78,79}$ Because of this mechanism of action and the absence of vasoconstrictor activity, lasmiditan is considered the first of the new drug class, the NAAMAs.

Lasmiditan has a high selectivity for the 5-HT1 $1_{F}$ receptors, which cannot be found on endothelial or smooth muscle cells of cerebral vessels. ${ }^{80}$ Moreover, its binding profile avoids potential AEs of the activation of $5-\mathrm{HT} 1_{B}$ receptors in peripheral blood vessels, particularly the coronary arteries. ${ }^{10,69}$ Based on its lack of vasoconstriction, this substance could serve as a treatment option for subjects with cardiovascular diseases to whom triptans are contraindicated.

\section{Expert opinion Conclusion}

Almost 30 years have passed since the introduction of triptans. They represented a major breakthrough in the acute treatment of migraine but are contraindicated in patients with previous cardio- and cerebrovascular diseases and in those with uncontrolled hypertension. Unfortunately, a high percentage of patients are not satisfied with this acute treatment, either for lack of response or for side effects. Since their introduction, no other acute drug class has passed all developmental stages.

Lasmiditan is a new effective drug with no vasoconstrictive action and a CNS-related AE profile.

There are some open questions that hopefully will find answers in future studies.

First of all, the serious matter of medication overuse headache $(\mathrm{MOH}){ }^{81}$ The pathophysiology of $\mathrm{MOH}$ is largely unknown, but both human and animal studies suggest modifications of the pain network. An excessive use of any of the acute drug classes (either specific or not) can cause headache to worsen and increase in frequency, and become less responsive to both acute and preventative medications. ${ }^{82}$ This leads to a vicious circle that is sometimes difficult to break. For triptans ( 5 -HT $1_{B} / 1_{D}$ agonists), the cutoff for the overuse is $\geq 15$ days/ month (for at least 3 months). Lasmiditan, as triptans, is a 5-HT agonist, but it differs for a unique chemical structure and for a high selectivity for the 5-HT1 $1_{\mathrm{F}}$ subtype. However, there is the possibility that overuse of lasmiditan will lead to $\mathrm{MOH}$.
A second important matter is the use of lasmiditan during pregnancy and breastfeeding. This concern is of paramount importance, thinking that migraine is more frequent in women and particularly during the fertile period of their life. Triptans do not seem to increase the rates of major congenital malformations, but children exposed to triptans in utero might have a higher risk of developing externalizing behaviors. ${ }^{17}$ They are considered probably compatible with breastfeeding. Lasmiditan, as triptans, is a high lipophilic molecule that in Phase II and III studies showed a safety profile different from the $5-\mathrm{HT} 1_{B} / 1_{D}$ agonists, with most of the AEs being related to CNS. This could limit their use during pregnancy and breastfeeding.

Future Phase III clinical trials will determine whether lasmiditan will become available in the market, and then active triptan comparator studies will assess patients' preference.

\section{Disclosure}

The authors report no conflicts of interest in this work.

\section{References}

1. Headache Classification Committee of the International Headache Society (IHS). The International Classification of Headache Disorders, 3rd edition (beta version). Cephalalgia. 2013;33(9):629-808.

2. Steiner TJ, Birbeck GL, Jensen R, Katsarava Z, Martelletti P, Stovner LJ. The Global Campaign, World Health Organization and Lifting the Burden: collaboration in action. J Headache Pain. 2011;12(3):273-274.

3. Bloudek LM, Stokes M, Buse DC, et al. Cost of healthcare for patients with migraine in five European countries: results from the International Burden of Migraine Study (IBMS). $J$ Headache Pain. 2012;13(5):361-378.

4. Steiner TJ, Birbeck GL, Jensen RH, Katsarava Z, Stovner LJ, Martelletti P. Headache disorders are third cause of disability worldwide. $J$ Headache Pain. 2015;16:58.

5. Steiner TJ, Stovner LJ, Vos T. GBD 2015: migraine is the third cause of disability in under 50s. J Headache Pain. 2016;17(1):104.

6. Negro A, Rocchietti-March M, Fiorillo M, Martelletti P. Chronic migraine: current concepts and ongoing treatments. Eur Rev Med Pharmacol Sci. 2011;15:1401-1420.

7. Becker WJ. Acute migraine treatment in adults. Headache. 2015;55(6):778-793.

8. Ferrari MD, Goadsby PJ, Roon KI, Lipton RB. Triptans (serotonin, 5-HT1B/1D agonists) in migraine: detailed results and methods of a meta-analysis of 53 trials. Cephalalgia. 2002;22(8):633-658.

9. Humphrey PP, Feniuk W. Mode of action of the anti-migraine drug sumatriptan. Trends Pharmacol Sci. 1991;12(12):444-446.

10. MaassenVanDenBrink A, Reekers M, Bax WA, Ferrari MD, Saxena PR. Coronary side-effect potential of current and prospective antimigraine drugs. Circulation. 1998;98(1):25-30.

11. Tfelt-Hansen P, Seidelin K, Stepanavage M, Lines C. The effect of rizatriptan, ergotamine, and their combination on human peripheral arteries: a double-blind, placebo-controlled, crossover study in normal subjects. Br J Clin Pharmacol. 2002;54(1):38-44.

12. MacIntyre PD, Bhargava B, Hogg KJ, Gemmill JD, Hillis WS. Effect of subcutaneous sumatriptan, a selective 5HT1 agonist, on the systemic, pulmonary, and coronary circulation. Circulation. 1993;87(2): 401-405.

13. Jayamaha JE, Street MK. Fatal cerebellar infarction in a migraine sufferer whilst receiving sumatriptan. Intensive Care Med. 1995;21(1):82-83. 
14. O'Connor P, Gladstone P. Oral sumatriptan-associated transmural myocardial infarction. Neurology. 1995;45(12):2274-2276.

15. Abbrescia VD, Pearlstein L, Kotler M. Sumatriptan-associated myocardial infarction: report of case with attention to potential risk factors. $J$ Am Osteopath Assoc. 1997;97(3):162-164.

16. Tepper SJ, Millson D. Safety profile of the triptans. Expert Opin Drug Saf. 2003;2(2):123-132.

17. Negro A, Delaruelle Z, Ivanova TA, et al. Headache and pregnancy: a systematic review. J Headache Pain. 2017;18(1):106.

18. Lionetto L, Negro A, Casolla B, Simmaco M, Martelletti P. Sumatriptan succinate: pharmacokinetics of different formulations in clinical practice. Expert Opin Pharmacother. 2012;13(16):2369-2380.

19. Negro A, D'Alonzo L, Martelletti P. Chronic migraine: comorbidities, risk factors, and rehabilitation. Intern Emerg Med. 2010;5(Suppl 1):S13-S19.

20. Lionetto L, Borro M, Curto M, et al. Choosing the safest acute therapy during chronic migraine prophylactic treatment: pharmacokinetic and pharmacodynamic considerations. Expert Opin Drug Metab Toxicol. 2016;12(4):399-406.

21. Rolan PE. Drug interactions with triptans: which are clinically significant? CNS Drugs. 2012;26(11):949-957.

22. Eyre BLKD, Eadie MJ, van Driel ML, Ross-Lee L, Hollingworth SA Triptan use in Australia 1997-2015: a pharmacoepidemiological study. Acta Neurol Scand. 2017;136(2):155-159.

23. Tfelt-Hansen P, Steiner TJ. Over-the-counter triptans for migraine: what are the implications? CNS Drugs. 2007;21(11):877-883.

24. Cevoli S, D'Amico D, Martelletti P, et al. Underdiagnosis and undertreatment of migraine in Italy: a survey of patients attending for the first time 10 headache centres. Cephalalgia. 2009;29(12):1285-1293.

25. Lantéri-Minet M, Massiou H, Romatet S, Barba A, Lucas C, Allaf B. An instrument to assess patient perceptions of satisfaction with acute migraine treatment (EXPERT Study). Headache. 2011;51(4):590-601.

26. Tfelt-Hansen P, Olesen J. Taking the negative view of current migraine treatments: the unmet needs. CNS Drugs. 2012;26(5):375-382.

27. Lipton RB, Buse DC, Serrano D, Holland S, Reed ML. Examination of unmet treatment needs among persons with episodic migraine: results of the American Migraine Prevalence and Prevention (AMPP) Study. Headache. 2013;53(8):1300-1311.

28. Martelletti P. Acute treatment of migraine: quo vadis? Expert Opin Pharmacother. 2017;18(11):1035-1037.

29. Yang LP. Sumatriptan/naproxen sodium: a review of its use in adult patients with migraine. Drugs. 2013;73(12):1339-1355.

30. Syed YY. Sumatriptan/naproxen sodium: a review in migraine. Drugs. 2016;76(1):111-121.

31. Law S, Derry S, Moore RA. Sumatriptan plus naproxen for the treatment of acute migraine attacks in adults. Cochrane Database Syst Rev. 2016;4:CD008541.

32. Chessman AW. ACP Journal Club. Review: sumatriptan plus naproxen improves acute migraine more than placebo, sumatriptan, or naproxen Ann Intern Med. 2014;160(4):JC8.

33. Cady R, Banks J, Nett RB, et al. Multi-center comparison of response to a single tablet of sumatriptan $85 \mathrm{mg}$ and naproxen $500 \mathrm{mg}$ vs usual therapy treating multiple migraine attacks as measured by the completeness of response survey. Headache. 2011;51(6):961-970.

34. Edwards KR, Rosenthal BL, Farmer KU, Cady RK, Browning R. Evaluation of sumatriptan-naproxen in the treatment of acute migraine: a placebo-controlled, double-blind, cross-over study assessing cognitive function. Headache. 2013;53(4):656-664.

35. Landy SH, Cady RK, Nelsen A, White J, Runken MC. Consistency of return to normal function, productivity, and satisfaction following migraine attacks treated with sumatriptan/naproxen sodium combination. Headache. 2014;54(4):640-654.

36. Tullo V, Valguarnera F, Barbanti P, et al. Comparison of frovatriptan plus dexketoprofen $(25 \mathrm{mg}$ or $37.5 \mathrm{mg}$ ) with frovatriptan alone in the treatment of migraine attacks with or without aura: a randomized study. Cephalalgia. 2014;34(6):434-445.
37. Asadollahi S, Heidari K, Vafaee R, Forouzanfar MM, Amini A, Shahrami A. Promethazine plus sumatriptan in the treatment of migraine: a randomized clinical trial. Headache. 2014;54(1):94-108.

38. Volans GN. Absorption of effervescent aspirin during migraine. $\mathrm{Br} \mathrm{Med}$ J. 1974;4(5939):265-268.

39. Tokola RA, Neuvonen PJ. Effect of migraine attacks on paracetamol absorption. Br J Clin Pharmacol. 1984;18(6):867-871.

40. Derry CJ, Derry S, Moore RA. Sumatriptan (intranasal route of administration) for acute migraine attacks in adults. Cochrane Database Syst Rev. 2012;(2):CD009663.

41. Derry CJ, Derry S, Moore RA. Sumatriptan (rectal route of administration) for acute migraine attacks in adults. Cochrane Database Syst Rev. 2012;(2):CD009664.

42. Tepper SJ, Cady RK, Silberstein S, et al. AVP-825 breath-powered intranasal delivery system containing $22 \mathrm{mg}$ sumatriptan powder vs $100 \mathrm{mg}$ oral sumatriptan in the acute treatment of migraines (The COMPASS study): a comparative randomized clinical trial across multiple attacks. Headache. 2015;55(5):621-635.

43. Lipton RB, McGinley JS, Shulman KJ, Silberstein SD, Wirth RJ, Buse DC. AVP-825 (Sumatriptan Nasal Powder) reduces nausea compared to sumatriptan tablets: results of the COMPASS Randomized Clinical Trial. Headache. Epub 2017 Oct 16. doi:10.1111/head.13199.

44. Freitag FG. Sumatriptan needle-free subcutaneous (Sumavel(®) DosePro ${ }^{\mathrm{TM}}$ ) approved for the acute treatment of migraine, with or without aura, and cluster headaches. Expert Rev Neurother. 2011;11(4): 481-490.

45. Brandes JL, Cady RK, Freitag FG, et al. Needle-free subcutaneous sumatriptan (Sumavel DosePro): bioequivalence and ease of use. Headache. 2009;49(10):1435-1444.

46. Cady RK, Aurora SK, Brandes JL, et al. Satisfaction with and confidence in needle-free subcutaneous sumatriptan in patients currently treated with triptans. Headache. 2011;51(8):1202-1211.

47. Goldstein J, Smith TR, Pugach N, Griesser J, Sebree T, Pierce M. A sumatriptan iontophoretic transdermal system for the acute treatment of migraine. Headache. 2012;52(9):1402-1410.

48. Derry CJ, Derry S, Moore RA. Sumatriptan (oral route of administration) for acute migraine attacks in adults. Cochrane Database Syst Rev. 2012;(2):CD008615.

49. Schuster NM, Rapoport AM. Calcitonin gene-related peptide-targeted therapies for migraine and cluster headache: a review. Clin Neuropharmacol. 2017;40(4):169-174.

50. Voss T, Lipton RB, Dodick DW, et al. A phase IIb randomized, doubleblind, placebo-controlled trial of ubrogepant for the acute treatment of migraine. Cephalalgia. 2016;36(9):887-898.

51. Goldstein DJ, Roon KI, Offen WW, et al. Selective seratonin 1F (5-HT(1F)) receptor agonist LY334370 for acute migraine: a randomised controlled trial. Lancet. 2001;358(9289):1230-1234.

52. Wainscott DB, Krushinski JH Jr, Audia JE, et al. [3H]LY334370, a novel radioligand for the 5-HT1F receptor. I. In vitro characterization of binding properties. Naunyn Schmiedebergs Arch Pharmacol. 2005;371(3):169-177.

53. Ramadan NM. Acute treatments: some blind alleys. Curr Med Res Opin. 2001;17(Suppl 1):s71-s80.

54. Capi M, de Andrés F, Lionetto L, et al. Lasmiditan for the treatment of migraine. Expert Opin Investig Drugs. 2017;26(2):227-234.

55. Nelson DL, Phebus LA, Johnson KW, et al. Preclinical pharmacological profile of the selective $5-\mathrm{HT} 1 \mathrm{~F}$ receptor agonist lasmiditan. Cephalalgia. 2010;30(10):1159-1169.

56. Färkkilä M, Diener HC, Géraud G, et al. Efficacy and tolerability of lasmiditan, an oral 5-HT(1F) receptor agonist, for the acute treatment of migraine: a phase 2 randomised, placebo-controlled, parallel-group, dose-ranging study. Lancet Neurol. 2012;11(5):405-413.

57. CoLucid Pharmaceuticas, Inc. Food-Effect Study in Healthy Subjects 2017. Available from: https://clinicaltrials.gov/ct2/show/NCT02233 296?term=NCT02233296\&rank=1. NLM identifier: NCT00265317. Accessed November 7, 2017. 
58. CoLucid Pharmaceuticals, Inc [webpage on the Internet]. Securities and Exchange Commission on April 30, 2015. 2017. Available from: www.getfilings.com/sec-filings/150430/CoLucid-Pharmaceuticals-Inc_ S-1.A/. Accessed November 7, 2017.

59. Pilgrim A, Dussault B, Rupniak N, et al. COL-144, an orally bioavailable selective 5-HT1F receptor agonist for acute migraine therapy. Cephalalgia. 2009;29(1 Suppl):1-166.

60. Liefaard L, Drenth HJ, Pilgrim AJ, et al. Prediction of therapeutically effective dose of COL-144 based on relationship between plasma concentrations and headache response (poster). Cephalalgia. 2009;29 (1 Suppl):1-166.

61. CoLucid Pharmaceuticals, Inc [webpage on the Internet]. Details Phase 3 Development Strategy for Lasmiditan to Address Major Unmet Needs in Acute Migraine Therapy. 2017. Available from: https://www. biospace.com/article/releases/colucid-pharmaceuticals-inc-detailsphase-3-development-strategy-for-lasmiditan-to-address-major-unmetneeds-in-acute-migraine-therapy-/. Accessed November 7, 2017.

62. Ferrari MD, Färkkilä M, Reuter U, et al. Acute treatment of migraine with the selective 5-HT1F receptor agonist lasmiditan - a randomised proof-of-concept trial. Cephalalgia. 2010;30(10):1170-1178.

63. CoLucid Pharmaceuticals, Inc [webpage on the Internet]. Lasmiditan Compared to Placebo in the Acute Treatment of Migraine: (SAMURAI). 2017. Available from: https://www.clinicaltrials.gov/ct2/show/NCT02 439320?term=SAMURAI\&rank=3. NLM identifier: NCT02439320. Accessed November 7, 2017.

64. CoLucid Pharmaceuticals, Inc [webpage on the Internet]. Three Doses of Lasmiditan (50 mg, $100 \mathrm{mg}$ and $200 \mathrm{mg}$ ) Compared to Placebo in the Acute Treatment of Migraine (SPARTAN). 2017. Available from: https:// www.clinicaltrials.gov/ct2/show/record/NCT02605174?term=lasmidit an\&rank=1. NLM identifier: NCT02605174. Accessed November 7, 2017.

65. GLADIATOR CoLucid Pharmaceuticals, Inc [webpage on the Internet]. An Open-label, Long-term, Safety Study of Lasmiditan for the Acute Treatment of Migraine (GLADIATOR). 2017. Available from: https:// www.clinicaltrials.gov/ct2/show/NCT02565186?term=GLADIATOR\& rank=3. NLM identifier: NCT02565186. Accessed November 7, 2017.

66. CoLucid Pharmaceuticals, Inc [webpage on the Internet]. CoLucid Pharmaceuticals Announces Achievement of Both Primary and Key Secondary Endpoints in the SAMURAI Phase 3 Pivotal Trial of Lasmiditan in Migraine. 2018. [updated September 62016; cited May 14, 2017]. Available from: https://globenewswire.com/news-release/2016/09/06/869611/0/en/ CoLucid-Pharmaceuticals-Announces-Achievement-of-Both-Primaryand-Key-Secondary-Endpoints-in-the-SAMURAIPhase-3-Pivotal-Trialof-Lasmiditan-in-Migraine.html. Accessed November 7, 2017.
67. Pietrobon D, Moskowitz MA. Pathophysiology of migraine. Annu Rev Physiol. 2013;75:365-391.

68. Houston DS, Vanhoutte PM. Serotonin and the vascular system. Role in health and disease, and implications for therapy. Drugs. 1986;31(2):149-163.

69. Tfelt-Hansen P, De Vries P, Saxena PR. Triptans in migraine: a comparative review of pharmacology, pharmacokinetics and efficacy. Drugs. 2000;60(6):1259-1287.

70. Akerman S, Holland PR, Goadsby PJ. Diencephalic and brainstem mechanisms in migraine. Nat Rev Neurosci. 2011;12(10):570-584.

71. Charles A. Advances in the basic and clinical science of migraine. Ann Neurol. 2009;65(5):491-498.

72. Deen M, Correnti E, Kamm K, et al. Blocking CGRP in migraine patients - a review of pros and cons. J Headache Pain. 2017;18(1):96.

73. Benemei S, Cortese F, Labastida-Ramírez A, et al. Triptans and CGRP blockade - impact on the cranial vasculature. J Headache Pain. 2017; 18(1):103.

74. Negro A, Lionetto L, Simmaco M, Martelletti P. CGRP receptor antagonists: an expanding drug class for acute migraine? Expert Opin Investig Drugs. 2012;21(6):807-818.

75. Adham N, Bard JA, Zgombick JM, et al. Cloning and characterization of the guinea pig 5-HT1F receptor subtype: a comparison of the pharmacological profile to the human species homolog. Neuropharmacology. 1997;36(4-5):569-576.

76. Ma QP. Co-localization of 5-HT(1B/1D/1F) receptors and glutamate in trigeminal ganglia in rats. Neuroreport. 2001;12(8):1589-1591.

77. Visser WH, Jaspers NM, de Vriend RH, Ferrari MD. Chest symptoms after sumatriptan: a two-year clinical practice review in 735 consecutive migraine patients. Cephalalgia. 1996;16(8):554-559.

78. Bouchelet I, Cohen Z, Case B, Séguéla P, Hamel E. Differential expression of sumatriptan-sensitive 5-hydroxytryptamine receptors in human trigeminal ganglia and cerebral blood vessels. Mol Pharmacol. 1996;50(2):219-223.

79. Mitsikostas DD, Sanchez del Rio M, Moskowitz MA, Waeber C. Both 5-HT1B and 5-HT1F receptors modulate c-fos expression within rat trigeminal nucleus caudalis. Eur J Pharmacol. 1999;369(3):271-277.

80. Reuter U, Israel H, Neeb L. The pharmacological profile and clinical prospects of the oral 5-HT1F receptor agonist lasmiditan in the acute treatment of migraine. Ther Adv Neurol Disord. 2015;8(1):46-54.

81. Negro A, Martelletti P. Chronic migraine plus medication overuse headache: two entities or not? J Headache Pain. 2011;12(6):593-601.

82. Negro A, Curto M, Lionetto L, Guerzoni S, Pini LA, Martelletti P. A critical evaluation on $\mathrm{MOH}$ current treatments. Curr Treat Options Neurol. 2017;19(9):32.
Journal of Pain Research

\section{Publish your work in this journal}

The Journal of Pain Research is an international, peer reviewed, open access, online journal that welcomes laboratory and clinical findings in the fields of pain research and the prevention and management of pain. Original research, reviews, symposium reports, hypothesis formation and commentaries are all considered for publication.

\section{Dovepress}

The manuscript management system is completely online and includes a very quick and fair peer-review system, which is all easy to use. Visit http://www.dovepress.com/testimonials.php to read real quotes from published authors. 